\title{
Factores de riesgo en el desarrollo de úlceras de presión y sus implicaciones en la calidad de vida
}

\author{
Risk factors in the development of pressure ulcers and its implications in the \\ quality of life

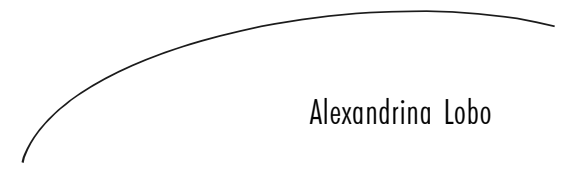

\section{Resumen}

En la vejez la incidencia de úlceras de presión (UPs) aumenta muy debido al aumento considerable del número de factores de riesgo. Entender su etiología es esencial para su prevención. En este contexto surge este estudio, debido a la necesidad de los autores identificaren que factores están implicados y son determinantes en el desarrollo de la úlcera de presión. También se analizó el efecto de las UPs en la percepción de la calidad de vida (CV). Objetivos: Determinar la contribución específica de cada factor de la escala de Braden en la predicción del desarrollo de UPs; explorar la hipótesis de que la ausencia de úlcera de presión se asocia a una mejor percepción de la CV e identificar que factores y condiciones están presentes en los ancianos con UPs. Material y Métodos: Estudio descriptivo, con una muestra aleatoria simple, constituída por 40 ancianos (12 con y 28 sin UPs) institucionalizados, para compararse la percepción de la calidad de vida y de los factores de riesgo. Se midieron: las medidas antropométricas, composición corporal, análisis sanguíneos, percepción de la calidad de vida y escala de Braden. Para el análisis estadístico se estableció la correlación existente entre las distintas variables con un nivel de significación (intervalo de confianza [1] del 95\%) p $<0,05$. Resultados: Una persona enferma con alteraciones en la nutrición tiene cerca de 4.5 veces más probabilidad de tener UPs y la presencia de alteraciones en la movilidad aumenta esta probabilidad en cerca de tres veces más. De las condiciones analizadas de la salud, se destacan las alteraciones hematológicas, designadamente la glucosa y el albumen. Al nivel de la composición corporal, el estado de hidratación es lo que
Palabras clave:

Anciano. Úlcera por Presión / etiología. Factores de Riesgo. Prevención Primaria. Calidad de Vida.

Institucionalización

Centro de Investigación de Actividad Física, Salude y Laser

Facultad de Ciencias del Deporte y Educación Física

Universidade do Porto, Porto, Portugal

Correspondencia / Correspondece

Alexandrina Lobo

Av. Padre Júlio Fragata, 109, 3.1 - San Victor

4700-413 Braga - Portugal

E-mail: damiaolobo@gmail.com 
tiene más significado estadístico. En lo general, la CV de estos ancianos es baja, siendo mayor en las mujeres $(25.6 \pm 11)$ y en el grupo de los ancianos sin UPs $(27 \pm 10)$. Conclusión: Una escala simplificada de tres factores (movilidad, nutrición y actividad) se asocia más al riesgo del desarrollo de UPs que la clásica escala de Braden.

\section{Resumo:}

Na velhice, a incidência de úlceras de pressão (UP) é muito maior devido ao considerável aumento no número de fatores de risco. Entender sua etiologia é fundamental para sua prevenção. Nesse contexto se insere o presente estudo, tendo em vista a necessidade de se identificar quais fatores estão implicados e são determinantes no desenvolvimento da UP. Também se analisou o efeito das UPs na percepção de qualidade de vida (QV). Objetivos: determinar a contribuição específica de cada fator da escala de Braden na predição do desenvolvimento da UP; explorar a hipótese de que a ausência de UP se associa a uma percepção melhor da QV e identificar que fatores e condições estão presentes nos idosos com UP. Material e métodos: estudo descritivo, com uma amostra aleatória simples, constituída por 40 idosos $(12 \mathrm{com}$ UP e 28 sem UP) institucionalizados, para se comparar a percepção da QV e dos fatores de risco. Foram avaliadas: as medidas antropométricas, a composição corporal, as análises sanguíneas, a percepção da QV e a escala de Braden. Para a análise estatística, estabeleceu-se a correlação existente entre as distintas variáveis com um nível de significância (intervalo de confiança [1] de 95\%) p < 0,05. Resultados: Uma pessoa enferma com alterações na nutrição tem cerca de 4,5 vezes mais probabilidades de ter UP, e a presença de alterações na mobilidade aumenta tal probabilidade cerca de três vezes mais. Das condições de saúde analisadas, destacam-se as alterações hematológicas, sobretudo a glicose e a albumina. Quanto à composição corporal, o estado de hidratação é o que possui mais significado estatístico. No geral, o nível de QV desses idosos é baixo, sendo maior entre as mulheres (25.6 \pm 11$)$ e no grupo de idosos sem UP $(27 \pm 10)$. Conclusão: Uma escala simplificada de três fatores (mobilidade, nutrição e atividade) se associa mais ao risco de desenvolvimento de UP que a clássica escala de Braden.

\section{Abstract:}

In old age, the incidence of pressure ulcers (UPs) increases a lot, due to the presence of several risk factors. Understanding its etiology is essential for its prevention. This study arises from the author's need to identify the factors involved and which determine the development of UPs. It also evaluates the effect of UPs in the perception of quality of life. Objective: To determine the specific contribution of each item of the scale of Braden in the prediction of the development of UPs; to explore the hypothesis that the absence of UPs is associated with one better perception of quality of life; and to identify what predisponent risk factors and conditions are present in elderly people with Ups. Materials and methods: Observational study on 40 institutionalized elderly (divided in 2 groups: 12 with and 28 without UPs) for comparison of the perception of the quality of life and risk factors. Measures: anthropometric ones, corporal composition, blood analyses, perception
Palavras-chave: Idoso. Úlcera de Pressão / etiologia. Fatores de Risco. Prevenção Primária. Qualidade de Vida. Institucionalização

Key words: Aged. Pressure Ulcer / etiology. Risk Factors. Primary Prevention. Quality of Life. Institutionalization 
of the quality of life, scale of Braden. Results: It was possible to conclude that a sick person with nutritional alterations is 4.5 times more probable to have UPs and to present alterations in mobility (probability is about three times higher). From the analyzed health conditions, blood alterations were established, mainly glucose and albumen. As for corporal composition, the state of hydration presented higher statistical significance. The quality of life among those elderly is low as a whole, being higher among men $(25.6 \pm 11)$ and in the group of elderly without UPs (27士10). Conclusion: A simplified 3-item scale (mobility, nutrition and activity) is more associated with the risk of development of UP than the classics scale of Braden.

\section{INTRODUTION}

El tratamiento de las UP es complejo, especialmente en ancianos. ${ }^{1}$ En los hospitales existen incidencias entre $2.7 \%$ y $29.5 \% .{ }^{2}$ Las UPs están asociadas a mayor mortalidad, pérdida de CV y aumento de los costos con los tratamientos. ${ }^{3}$ Los hospitales y las clínicas de reposo han puesto en ejecución escalas de evaluación del riesgo y protocolos de tratamiento, que permiten disminuir la incidencia y el predominio de las UPs. ${ }^{4}$

Muchos estudios demuestran que las UPs non son apenas consecuencia de la existencia de presión. La malnutrición, la piel mojada, las alteraciones en movilidad y percepción, la linfopenia y los niveles bajos de albumen sérica y de hemoglobina son factores significativos de riesgo para el desarrollo de UPs en ancianos que están confinados a la cama y a la silla. ${ }^{5} \mathrm{No}$ existen exámenes específicos para el diagnóstico de UPs. De cualquier manera, existen más de 100 productos para el tratamiento de UPs. Si bien que productos elegidos para su tratamiento de manera inadecuada pueden causar daños en las heridas y/o retrasar la curación. La gente enferma con las heridas exige mayor ingestión de proteínas y calorías para facilitar la regeneración tecidular al nivel celular.

La investigación también sugiere que un suplemento de multivitaminas, designadamente vitamina C, A, E y zinc, así como hierro, ayudan a estimular la cicatrización. ${ }^{6}$ Por ejemplo, la carencia de proteínas (hipoalbuminémia) daña la angiogénese, la proliferación de los fibroblastos, la síntesis y la función del colagéneo y la contestación del sistema inmunitario; está demostrado que algunos aminoácidos, como la arginine y la glutamina, son estimulantes de la cicatrización. Una dieta rica en carbohidratos todavía facilita la cicatrización, habiendo también necesidad de ácidos gordos esenciáis. Mientras que la vitamina A y el complejo B existen habitualmente en cantidad suficiente en la alimentación, ya el suplemento de vitamina $C$ es necesario para la síntesis y la maduración del colagéneo, y la vitamina $\mathrm{D}$ es necesaria para la absorción intestinal de calcio. En lo que respecta a los minerales/oligoelementos, el zinc tiene una función estimulante de los neutrófilos, su carencia se expresa en un aumento de la susceptibilidad a la infección y en una cicatrización retrasada. El 
hierro es necesario para la síntesis del heme y por lo tanto básico para una buena oxigenación de las telas. También intervienen numerosas enzimas y los que son sus cofactores también son imprescindibles (ejemplo, el cobre). ${ }^{7}$

La desnutrición, cuando está asociada a la deshidratación, tornase un importante factor de riesgo por reducir la elasticidad de la piel. Cuanto más activa e independiente la persona enferma, menor es el riesgo de la formación de UP y mayores las posibilidades de cicatrización. El factor clave relacionado con la cicatrización es el retiro de los factores causadores. Independientemente del tratamiento prescrito para los cuidados a la herida, si la presión no es aliviada, por la persona enferma o por la enfermera, no habrá cicatrización.

En este estudio procedemos a la evaluación de la calidad de vida para proporcionar información valiosa referente al funcionamiento físico, mental y social del individuo, ${ }^{8}$ siendo un indicador recomendado para el $\mathrm{WHO}^{9}$ para la evaluación del estado de la salud de las poblaciones. La opinión que la persona tiene de los factores determinativos de su salud viene siendo considerada como coadyuvante de indicadores tradicionales en la evaluación de necesidades en salud, en vista de sí mismo que las interacciones físicas, emocionales y sociales complejas están implicadas en el desarrollo de comportamientos y de los estilos de vida que promueven la calidad de vida.

Este trabajo tiene la intención de acercar, desde una perspectiva científica, la relación entre los diversos factores de riesgo para las UP y su desarrollo, que por sus especiales características requiere una abordaje multidisciplinar, si bien que han sido identificado principalmente como un foco de alta sensibilidad para los cuidados del oficio de la enfermera. Las normas de práctica de cuidados aceptos a nivel internacional se han repasado cuánto a la manera de actuar en las UP y es este sentido que investigamos para contribuir con datos relevantes para la práctica clínica, en este ya tema discutido de par en par.

\section{MATERIAL Y MÉTODOS}

\section{Diseño del estudio}

Esto es un estudio descriptivo con el objetivo de identificar las causas o los factores posibles del riesgo que se pueden implicar más o menos en el acontecimiento UPs. Intentamos abordalo con datos concretos como la composición corporal, datos laboratoriales, estado nutricional y enfermedades asociadas. Importa salvaguardar que los cuidados prestados en las diferentes instituciones son uniformes y están de acuerdo con las recomendaciones internacionales. En este estudio participaran 95 ancianos, con escala de Braden inferior o igual a 17 puntos, de 5 instituciones del ayuntamiento de Porto y de Braga. Fue utilizada la técnica de muestra estratificada. Se buscó determinar la relación existente entre cada factor que constituye la escala de Braden y el resultado relativo de la presencia o no de UPs y saber las implicaciones de las úlceras de presión en la calidad de vida. ${ }^{10}$ 


\section{Sujetos y Medidas}

En julio de 2007, 40 ancianos (28 mujeres y 12 hombres) han sido seleccionados y invitados a participar en el estudio. La pesquisa fue concluída dentro de los padrones exigidos por la Declaración de Helsinki y aprobada por la comisión de ética de las instituciones. Todos habían firmado el asentimiento informado, de la libre y clarificada voluntad demostraron entender los objetivos del estudio. Los criterios de exclusión fueron ancianos con necesidad de oxígeno, agitación y Mini-Mental State Examination inferior a 24 puntos. ${ }^{11} \mathrm{El}$ peso fue obtenido a través de una balanza digital marca Seca 708, con aproximaciones a las centésimas. La altura fue evaluada entre o vertéx (punto arriba de la cabeza, en el plano mediano-sagital) y el plano de referencia del suelo. El índice de masa corporal (IMC) fue calculado a través del peso/altura2 (en kg/ $\mathrm{m} 2$ ). En que: $<18,4$ es delgado; entre 18,5 y 24,9 es normal, arriba de 25 es exceso de peso, donde $>30$ es obesidad. El Bodystat QuadScan 4000 fue utilizado para analizar la composición corporal, por ser un método simple y no invasor de evaluación de la composición corporal, con la impedancia bioeléctrica. $^{12}$

Este dispositivo es usado para medir la grasa del cuerpo y la hidratación a través de una cadena eléctrica de la intensidad reducida, esa la cubre, estando sin embargo muy bajo e imperceptible para el ciudadano. Fue llevado junto con las cosechas para el análisis sanguineo, después de $12 \mathrm{~h}$ del ayuno y evaluó la tensión arterial. Los valores de los adoptados de la referencia son los siguientes: glucosa $(70-100 \mathrm{mg} / \mathrm{dl})$, albumen $(>3,3 \mathrm{~g} / \mathrm{dl})$,

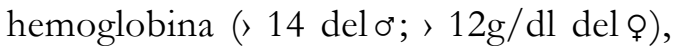
linfócitos (1.5-3.5\%), colesterol ( $200 \mathrm{mg} / \mathrm{dl})$, presión arterial sistólica $(140 \mathrm{mmHg}) \mathrm{y}$ presión arterial diastólica ( $85 \mathrm{mmHg}$ ). Para la grasa del cuerpo, los valores de la referencia para los varones están entre $17-21 \%$ y para las mujeres entre $22-31 \%$, y para la hidratación los valores de la referencia para los varones están entre $55-65 \%$ y para las mujeres entre $50-60 \%$.

La concentración de albumen sérica depende de muchos factores, pero hay limitaciones causadas por la vejez que intervienen en la detección del estado nutricional. Sin embargo, está descrita una fuerte correlación entre los niveles de albumina sérica con aumentos en la morbilidad (tiempo de la internación, cicatrización deficiente de heridas) siendo uno del los más frecuentes factores usados como componente pronóstico de los índices y la predicción de las complicaciones. ${ }^{13,14}$ El SF-36 fue utilizado para medir la percepción de la CV, siendo una medida genérica de salud, una vez que se destina a medir conceptos de salud que representan valores humanos básicos relevantes a la funcionalidad y al bienestar de cada uno. Presenta ocho dimensiones: salud general (SG), función física (FF), salud mental (SM), función social (FS), vitalidad (VT), dolor corporal (DC), cambios en la salud (MS), desempeño físico (DF) y desempeño emocional (DE). En cada dominio los scores varian entre 0 (menos salud) y 100 (más salud) [2]. La escala de Braden fue aplicada para identificar a la gente enferma con riesgo elevado para convertirse 
sube (en este estudio envejecidos con menos se consideran que 17 puntos). ${ }^{10,15}$ Las puntuadas de las áreas en este instrumento, ajustado específicamente según esta población, incluyen los niveles de la opinión sensoria de la persona enferma, su movilidad, nutrición, actividad, tan bien como la presencia o la ausencia de la humedad, de la fricción y del agotamiento. Éstas son puntuadas de 1 a 4 y se calculan el total, cuánto menos la puntuación, mayor el riesgo de la persona enferma para sufrir la solución de continuidad en la piel.

La clasificación del sube definido para la úlcera de presión fue utilizada (1997) de acuerdo con su estadio (grado 1.2.3 o 4), la localización (sacro, trocanteres, calcaneos, etc.) y el tamaño (regla con acercamiento al centímetro, en registro irregular de las heridas en una dimensión más grande). El sistema de clasificación a través la "descripción visual" intenta ser una representación exacta de la forma y extensión de las lesiones. ${ }^{16}$

Así, para la determinación del área y del volumen,fue seleccionada la fórmula matemática de Kunding, ${ }^{17}$ para ser más funcional (anchura x 0.785 de la longitud $\mathrm{x}$ para la anchura y la profundidad x 0.327 del área x para el volumen).

Los problemas de salud habían sido asociados de los registrados (diabetes, hipertensión arterial, fiebre cardiaca, problemas y enfermedad vascular periférica) y medicación depresiva del SNC (analgesia, tranquilizantes) y del vasoconstritora, una época que se describe en literatura como factores predisponientes potenciales. ${ }^{4,18}$ Para la evaluación nutricional se utilizó la Mini Evaluación Nutricional (MAN) que fue obligada y convertida para evaluar el riesgo de la desnutrición en ancianos y identificar los que pueden beneficiarse de una intervención precoz. ${ }^{13,19}$ En ancianos con úlceras de presión, las necesidades de energía aumentan y varían de acuerdo con el grado de las UPs, correspondiendo a más 30/40 Kcal./Kg./día y al aumento del nivel proteínico de $1 / 2 \mathrm{~g} / \mathrm{Kg}$./ día. $^{6}$

\section{Cuidados de Enfermería en los} Ancianos con Braden $\leq 17$

El tratamiento en las instituciones está de acuerdo con las directrizes del Royal College of Nursing, ${ }^{20}$ incluso medidas para promover la percusión y oxigenación de los tejidos: reducción y/o eliminación de fricciones y el alivio de la presión a través de la alternancia de decúbitos (movilizaciones mínimas de 4 en $4 \mathrm{~h}$ evitando os decúbitos de las zonas afectadas y con transferencia para la silla después de los cuidados de higiene y por la tarde, conforme tolerancia), usando superficies y/o dispositivos de alivio de presión (almohadas). Manteniendo la piel suave y elástica a través de aceite y controlando la incontinencia y la humedad cerca de la piel. ${ }^{4}$ Las intervenciones destinadas para controlar la sintomatología de la enfermedad crónica que afecta el tegumento saludable había consistido esencialmente en la regulación de la medicación a la subsistencia del interior, los niveles de la glucosa de los límites normales, 
uso de la técnica aséptica y las precauciones estándares para la prevención de la infección, haciendo los registros diarios de las señales y de los síntomas identificados para supervisar.

\section{Análise estadística}

Para el tratamiento estadístico de los datos, se utilizou el programa SPSS (Statistical Package for the Social Sciences), versión 13, con un nível de significancia de 5\%. En una primera etapa, los datos fueron explorados a través de estadística descritiva, nomeadamente medidas de tendencia central y de dispersión. Se utilizó el T-teste para comparar los géneros y la correlación de Pearson para verificar la existencia de asociaciones entre las diferentes variables. Uno calculaba el porcentaje medio de la ocurrencia de las condiciones y de los factores predisponientes del riesgo. Para la descripción de la relación entre los factores de riesgo y la existencia de úlceras de presión se utilizó el risco relativo (RR), que es la razón de la incidencia en individuos con los factores de riesgo (Braden $\leq 17$ ) y de la incidencia en individuos sin factores de riesgo. Las probabilidades $(\mathrm{OR}=$ Odds Rácio $)$ permiten evaluar el riesgo del desarrollo de úlceras de presión en ancianos, de acuerdo con sus factores de riesgo.

\section{RESULTADOS}

Los índices de la incidencia de úlceras verificadas de la presión en la población de riesgo en este estudio $(1.7 \%)$ son inferiores a los resultados de estudios internacionales y nacional. ${ }^{21,22}$ Los datos del programa INTERREG III-B divulgan que este es un problema que afecta cerca del $12 \%$ de la población acamada (sea en instituciones, sea en sus residencias). En el servicio de Ortotraumatologia del Hospital Coimbra, la incidencia estaba de $2.47 \%$ de 1997 después de un trabajo desarrollado con el objetivo de reducir estos niveles. El servicio de mujeres de la medicina del hospital de Funchal era no más de $3.1 \%$ cuando estaba determinado en la población presentado por lo menos un factor de riesgo. ${ }^{23}$ En el centro regional de la salud de Funchal, el predominio de la gente enferma con UP fue $7.1 \%$ del hospital y el $3.9 \%$ en hogares y servicios de la internación de duración larga. De los 193 enfermos con úlceras, fueran encontradas 439 UPs en varios estadios, donde predominaba el estadio II.

Las localizaciones más frecuentes habían estado al trocanter (el 29\%), a los sacros de la región (el $24 \%$ ) y al calcaneo (el 15\%). ${ }^{24}$ En nuestro estudio, el $31.8 \%$ del sube se sitúan en sacro, otros el $31.8 \%$ en trocanteres y el $18 \%$ en los calcaneos, estando en el $48 \%$ de los casos del grado 2 , en $22.7 \%$ del grado 3 y el $30 \%$ de los casos del grado 4 .

Es sin duda alguna que los factores intrínsecos del riesgo son los que predisponen el individuo al desarrollo de sube, siendo más importantes los que comprometen movilidad y la percusión tecidular. La incapacidad de si mover libremente implica que el individuo no es capaz de aliviar la presión. También el déficit neurológico puede reducir la movilidad en cuanto a la sensibilidad al dolor (ejemplo: diabético con neuropatías periféricas). Ya 
Dealey ${ }^{25}$ discute que la pérdida de sensibilidad sea de preocupación que la limitación en movilidad. La característica envejecida con sensibilidad guardada obtiene para solicitar la ayuda cuando siente malestar, mientras que cuando ha enganchado la sensibilidad tolera las presiones dibujadas hacia fuera con la formación consiguiente de sube. La escala de Braden es asociada (que no establece sin embargo relaciones de causalidad), de la manera significativa estadística con todo el artículo que lo compone, según lo representado en la tabla. La correlación es distinguida para $\mathrm{p}=0.01$, incorpora el resultado de la escala de Braden y la movilidad más fuerte del artículo para ser (al lado de 1) y positivo, indicando que en promedio, cuando la movilidad aumenta también la cuenta de los aumentos de Braden, se disminuye que el riesgo a convertirse sube. ${ }^{10}$ También la nutrición y la actividad correlacionanse de manera significativa estadística para un $\mathrm{p}=0.01$; siguiente para $\mathrm{p}=0.05$, la humedad $(\mathrm{P}=0.604)$ y la opinión sensible $(\mathrm{P}=0.547)$.

La tabla 2 permíte analizar la relación entre los diversos factores de la escala de Braden y la existencia de UP. Uno evidencia eso para $\mathrm{p}=0.05$ que la regresión logística es significativa para la nutrición $(\mathrm{OR}=4.571)$ y para la movilidad $(\mathrm{OR}=3.027) \cdot{ }^{10}$ Se puede concluir que una persona enferma con alteraciones en la nutrición tiene cerca de 4.5 veces más probabilidad de tener UP y la presentación de alteraciones en movilidad tiene cerca de 3 veces más probabilidad de tener UP. Ya Perneger et al. ${ }^{26}$ sugieren en un estudio con 2.373 enfermos hospitalizados los factores más importantes de la escala de Norton para la predicción del desarrollo de UP son la actividad y la movilidad.

Tabla 1 - Correlación de Pearson de los factores de la escala de Braden. Braga, Portugal, 2007.

\begin{tabular}{lcccc}
\hline & Humedad & Movilidad & Nutrición & Braden \\
\cline { 2 - 5 } Percepcione sensitiva & .023 & $.312\left(^{*}\right)$ & .252 & $.547\left(^{*}\right)$ \\
Humedad & 1 & $.465\left(^{* *}\right)$ & .066 & $.604\left(^{*}\right)$ \\
Actividad & $.370(*)$ & $.397\left(^{*}\right)$ & -.082 & $.526\left(^{* *}\right)$ \\
Movilidad & $.465(* *)$ & 1 & .310 & $.728\left(^{* *}\right)$ \\
Nutrición & .066 & .310 & 1 & $.536(* *)$ \\
Fricción & -.018 & -.084 & $.428(* *)$ & $.357\left(^{*}\right)$ \\
\hline
\end{tabular}

* Correlación significativa para $\mathrm{P}=0.05$

** Correlación significativa para $\mathrm{P}=0.01$ 
Tabla 2 - Regresión Logística de los factores de la escala de Braden. Braga, Portugal, 2007.

\begin{tabular}{lccc}
\hline & OR & \multicolumn{2}{c}{ IC (95\%) } \\
\cline { 2 - 4 } & & Mínimo & Máximo \\
\cline { 2 - 4 } Percepción sensitiva & .097 & .935 & 1.628 \\
Humedad & .095 & .008 & 1.130 \\
Actividad & .574 & .097 & 3.397 \\
Movilidad & $3.027^{*}$ & .125 & 13.607 \\
Nutrición & $4.571^{*}$ & .270 & 17.482 \\
Fricción & .041 & .002 & .769 \\
\hline
\end{tabular}

* Significativa $\mathrm{P}=0.05$

La edad es resaltada, para la mayoría de los autores, como uno de los factores más relevantes en la fisiopatogenese de las úlceras de presión, asociada a las alteraciones decurrentes del envejecimiento. ${ }^{22,27}$ En esta muestra, los pacientes con UPs presentan una edad media de 78.3 años, inferior a quién también presenta riesgo pero no desenvolvió UPs con 82.9 años sin diferencias significativas estadísticas (tabla 3).

El IMC mínimo fue de 18.8 y el máximo de 31.8 y en media $23.4 \pm 3.1$ sin diferencias significativas entre los sexos, ni entre los grupos. Sin embargo, debese reconocer las deficiencias ocultas de los nutricionais del poder del exceso de peso. Los bioquímicos de las medidas en fecha el albumen del sérica, la hemoglobina, el colesterol y la glucosa son ventajosos, teniendo en la consideración el estado del nutricional de la persona enferma. En lo cuadro 1 los valores de estos parámetros de los laboratoriais para los 2 grupos se representan. La correlación de la escala de Braden con el glicemia es distinguida en el promedio para $\mathrm{p}=0.05,(\mathrm{P}=-0.671)$, indicando que, cuando la glicemia aumenta la cuenta de Braden disminuida, aumentase el riesgo para desenvolver úlceras de presión. Seguidamente, la hidratación $(\mathrm{P}=0.631)$ y la albumen $(\mathrm{P}=0.587)$ indican que en medio, cuando aumentan también la cuenta de Braden, por lo tanto disminuye el riesgo del desarrollo de UPs. Una evidencia que la diferencia entre los grupos es significativa para la glucosa, el albumen y el linfocitose. En cuanto al porcentaje de hidratación, esta tiene diferencia significativa estadística, siendo el grupo con UPs con mayor riesgo de la deshidratación (52.1 \pm 2.3$)$. La grasa corporal aparece con valores inferiores en el grupo sin UPs (14.2 \pm 2.7$)$, cual es de acuerdo con los valores del albumen sérico $(2.9 \pm 0.9)$, dada a la relación entre estos dos parámetros. ${ }^{10}$

El predominio de la mala nutrición en ancianos institucionalizados es cambiable de acuerdo con la metodología y el tiempo de institucionalización, la colocación introduce 19 y $\operatorname{los} 60 \%{ }^{28}$ Estos valores son bastante 
significativos para hacer frente a la mala nutrición como factor que causen diferenciación en la evolución de la cicatrización en este grupo de ancianos.

Nuestros resultados relativos a la ingestión nutricional sugieren que llegue en puerto que proteína (obtenido para una alimentación rica en carne, pescados, huevos) es escasa ( $0.7 \mathrm{~g} /$ $\mathrm{kg} /$ diámetro) en el grupo con sube, teniendo adentro considere las necesidades de cicatrización (aumento 1 el $2 \mathrm{~g} / \mathrm{kg} /$ diámetro).
El riesgo nutricional en envejecidos con sube es significativo estadísticamente $(\mathrm{P}=0.776)$, demostrando que eso es capaz predecir el riesgo de los asociados de las complicaciones las enfermedades. ${ }^{13,19}$ Particularmente la deuda, los factores se relacionaron con las alteraciones fisiológicas y sociales, la ocurrencia de la enfermedad crónica, el uso de algunas medicaciones, los problemas en la alimentación (comprometiendo la masticación y la deglutición) y las alteraciones de la movilidad con la dependencia funcional. ${ }^{29}$

Tabla 3 - Características de la amostra por Sexo y Grupo. Braga, Portugal, 2007.

\begin{tabular}{lcccc}
\hline Braga, Portugal, 2007. & \multicolumn{2}{c}{ Sexo } & \multicolumn{2}{c}{ Grupo } \\
\hline & Mujeres & Varones & Con UP & Sin UP \\
\cline { 2 - 5 } Idad (años) & $83.5 \pm 8.6$ & $76.8 \pm 8.8$ & $78.3 \pm 9.2$ & $82.8 \pm 8.8$ \\
IMC & $22.8 \pm 2.7$ & $24.8 \pm 3.4$ & $24 \pm 3.3$ & $23.1 \pm 3$ \\
Braden & $11.5 \pm 1.7$ & $11.3 \pm 2.9$ & $11.2 \pm 3.2$ & $11.5 \pm 1.4$ \\
Funcione Física (\%) & $10.9 \pm 10.9$ & $13.8 \pm 12$ & $7.9 \pm 11.2$ & $13.4 \pm 11$ \\
Desempeño Físico (\%) & $8.6 \pm 9.7$ & $11.2 \pm 9.4$ & $7.7 \pm 8.7 *$ & $10 \pm 10^{*}$ \\
Desempeño Emocional (\%) & $36.2 \pm 27.9$ & $34.9 \pm 22.9$ & $21.8 \pm 21.6$ & $41.8 \pm 26$ \\
Câmbios en la Saúde (\%) & $26.8 \pm 19.2$ & $25.0 \pm 23.8$ & $18.8 \pm 21.7$ & $29.5 \pm 19.3$ \\
Dolor corporal (\%) & $47.5 \pm 20.9$ & $38.8 \pm 23.1$ & $37.7 \pm 24.3$ & $48 \pm 20$ \\
Salude General (\%) & $25.7 \pm 10.4$ & $29.1 \pm 12.4$ & $25.4 \pm 11.7$ & $27.3 \pm 10.8$ \\
Funcione Social (\%) & $12.1 \pm 15$ & $16.3 \pm 13$ & $16.9 \pm 17$ & $11.8 \pm 13.2$ \\
Salude Mental (\%) & $44.1 \pm 25.4$ & $50.7 \pm 21.6$ & $42.3 \pm 24.4$ & $47.7 \pm 24.5$ \\
Vitalidad (\%) & $11.7 \pm 13.8$ & $10.6 \pm 9.3$ & $8.9 \pm 11.5$ & $12.4 \pm 13$ \\
\hline
\end{tabular}

*T-teste significativo $\mathrm{p}=0.05$

Clínico, las enfermedades bajas se asocian predominantemente en ambos los grupos del estudio, punto con respecto a los que comprometan el sistema cardiovascular, el HTA en $35.7 \%$ envejecidos del grupo fuera suben más específicamente y el $25 \%$ en que presentan cardiaco suben y otros problemas en $16.7 \%$ envejecidos, yendo a la reunión de lo que él señala a autores españoles. ${ }^{30} \mathrm{La}$ perfusión deficiente del oxigénio en telas puede resultar de problemas cardiacos, de enfermedad vascular periférica, de la diabetes o de la ingestión de los vasoconstritores de las medicinas. 
En cuanto a las medicinas usadas, principalmente del uso continuo, que puede también contribuir para el desarrollo de úlceras de presíon, los anti-hipertensores son utilizados por el $31 \%$ de los ancianos (teniendo implicaciones en la reducción del flujo sanguíneos y la perfusión tecidular, haciendolos susceptibles a la presión), mientras que los sedativos y las analgesias dañan la movilidad, siendo utilizados para las 57\% envejecidas. ${ }^{31}$

El tiempo medio de la institucionalización, como otro aspecto importante en el origen de muchas alteraciones o complicaciones en pacientes hospitalizados, fue menor para los pacientes con UPs (7.7 \pm 3.7 ), comparativamente a los sin UPs

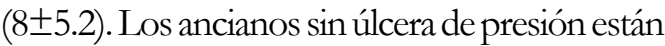

insertados en las cuentas más levantadas deBraden y tenían mejor percepción de la calidade de vida, excepto en la dimensión de la FS, con qué estará asociada a una gran proximidad del contacto exigida por la necesidad de cuidados más frecuentes en los ancianos con úlcera de presión. En relación a la DC, es sorprendente que el grupo sin úlcera de presión $(48 \pm 20 \%)$ tiene un valor superior al grupo con úlcera de presión $(37,7 \pm 24 \%)$, siendo razonable que el dolor estaba bien controlado en los ancianos con UPs.

En la generalidad, la CV de éstos ancianos es baja, siendo levemente superior en los varones $(25.6 \pm 11)$. Las mujeres $(24.9 \pm 10)$ presentan mejores resultados en las dimensiones DE, MS, DC y VT.

Cuadro 1 - Correlación de Pearson y Médias por grupos de los factores de risco de UP. Braga, Portugal, 2007.

\begin{tabular}{|l|l|c|c|c|}
\hline \multirow{2}{*}{} & & Pearson para E. & \multicolumn{2}{|c|}{ Grupos } \\
\cline { 3 - 5 } & & Braden & Con UPs & Sin UPs \\
\hline \multirow{4}{*}{$\begin{array}{l}\text { Datos } \\
\text { Bibliográficos }\end{array}$} & Proteínas $(\mathrm{g} / \mathrm{dl})$ & .341 & $0.52 \pm 0,3$ & $0.6 \pm 1.2$ \\
\cline { 2 - 5 } & Glucose $(\mathrm{mg} / \mathrm{dl})$ & $-.671^{* *}$ & $129 \pm 2.5^{*}$ & $98 \pm 1.1^{*}$ \\
\cline { 2 - 5 } & Colesterol $(\mathrm{g} / \mathrm{dl})$ & .361 & $201 \pm 1.3$ & $203 \pm 0.8$ \\
\cline { 2 - 5 } & Albumen $(\mathrm{g} / \mathrm{dl})$ & $.587^{* *}$ & $2.9 \pm 0.9^{*}$ & $3.5 \pm 1.2^{*}$ \\
\cline { 2 - 5 } & Hemoglobina $(\mathrm{g} / \mathrm{dl})$ & .545 & $12 \pm 1.4$ & $13 \pm 2.2$ \\
\cline { 2 - 5 } & Linfócitos $(\%)$ & .513 & $3.9 \pm 1.2^{*}$ & $2.3 \pm 1.8^{*}$ \\
\hline \multirow{3}{*}{$\begin{array}{l}\text { Composición } \\
\text { Corporal }\end{array}$} & Grasa corporal $(\%)$ & .428 & $14.2 \pm 2.7$ & $16.3 \pm 1.5$ \\
\cline { 2 - 5 } & Hidratación corporal $(\%)$ & $.631^{* *}$ & $52.1 \pm 2.3^{*}$ & $57.4 \pm 3.1^{*}$ \\
\hline \multicolumn{2}{|l|}{ Mini Evaluación Nutricional (puntos) } & $.776^{* *}$ & $16.2 \pm 1.2^{*}$ & $19.5 \pm 1.1^{*}$ \\
\hline Ingestión Proteínas $(\mathrm{g} / \mathrm{Kg} /$ dia) & .435 & $0.7 \pm 0.01$ & $1.1 \pm 0.1$ \\
\hline \multicolumn{2}{|l|}{ Tensión Arterial $(\mathrm{mmHg})$} & .287 & $141 / 90$ & $138 / 88$ \\
\hline
\end{tabular}

* T-teste, estatisticamente significativo $\mathrm{p}=0.05$

** Pearson, estatisticamente significativo $\mathrm{p}=0.05$ 
Existen muchos sistemas disponibles de clasificación, pero ninguno es totalmente inclusivo. En este contexto aparecen escalas de la evaluación del riesgo que permiten identificar y cuantificar los factores de riesgo. La escala de Braden se ha aplicado de par en par y nuestros resultados permiten afirmar que está sobre un instrumento sensible y válido. Sin embargo exige los entrenamientos, severidad de parte del tasador en su uso. En este estudio fue observado que otros factores, tales como la glucosa, el albumen, la grasa corporal y los linfocitos son esenciales de predecir que el riesgo del desarrollo de úlceras de presión.

Analizando el riesgo de desarrollo de úlceras de presión, fue posible establecer una relación entre los factores de riesgo analizados.

\section{CONCLUSIÓN}

El mandato social del oficio de enfermera entiende la promoción de la salud, la prevención de la enfermedad y del blanqueo enjaezando la salud del cliente..$^{20} \mathrm{La}$ evaluación del riesgo se debe considerar como un proceso dinámico, dado que la condición de los sujetos no es una condición estática. Probablemente si todos los esfuerzos son canalizados en la dirección de prevenir el desarrollo de úlceras de presión, no sólo se conseguirán cuidados mejores así como una mejor calidad de vida para un número más grande de ancianos.

Es una situación que interviene grandemente con los afectados envejecidos, según lo presentado en el cuadro 2. Para contribuir a una educación mejor para la salud envejecidas, divulgaremos nuestros resultados y sugerencias. El desarrollo de este trabajo traerá las implicaciones importantes para la práctica de oficio de enfermera, nominado, en lo que dice respecto al tratamiento del sube.

La sugerencia es que los estudios de la incidencia y del predominio tendrían que ser periódicamente llevados, para el monitorización y la integración de datos dentro de una política local encendido sube. Ese tipo de resultado permite medir la calidad de los cuidados en cada institución.

Ese estudio permitió concluir: (i) los ancianos institucionalizados evaluados presentan exceso de peso; (ii) el sexo femenino presenta una percepción de calidad de vida más baja que el sexo masculino; (iii) los ancianos entre si presentan niveles de ApF muy diferentes; (iv) el nivel de dependencia de los ancianos es grave para $29,2 \%$ y $60 \%$ son moderadamente dependientes; (v) la edad presenta una relación linear con el grado de dependencia (Pearson=0,045); (vi) cuanto mayor el peso, mayor el grado de dependencia (Pearson=0,343); (vii) el número de enfermedades crónicas está inversamente asociado al nivel de funcionalidad en las AVD's $($ Pearson $=-0,260)$ y al IMC (Pearson $=$ 0,285). La presencia de UP puede provocar un cambio en las actividades de la vida diaria, teniéndo en cuenta los problemas físicos, emocionales o sociales que si puede traducir en un déficit de la auto-estima, de la autonomia y de la alteración de la imagen corporal. 


\section{AGRADECIMIENTOS}

A las instituciones que autorizaran la colecta de datos y en especial a mis compañeras: Enf ${ }^{a}$
Rafaela, Enfa Sandra y restantes miembros de los equipos de cuidados, por el apoyo prestado, a todos muchas gracias.

\section{BIBLIGRAFIA}

1. Boni-Saenz A, Dranove D, Emanuel L, Sasso AL. The price of palliative care: toward a complete accounting of costs and benefits. Clin Geriatr Med 2005; 21: 147-63.

2. Gerson L. The incidence of pressure sores in active treatment hospitals. Int J Nurs Stud 1975; 12: 201-4.

3. Lyder C. Pressure ulcer prevention and management. JAMA 2003; 289: 223-6.

4. Stephens F, Bick D. Guidelines: a pressure ulcer risk assessment and prevention audit. Nursing Management 2002; 9: 3-14.

5. Thomas D. Improving outcome of pressure ulcers with nutritional interventions: a review of the evidence: Nutrition 2001; 17: 121-5.

6. Mateus C. A nutrição no tratameno de feridas. 2004. Disponível em: URL: http:// www.gaif.net/artigos-rev.htm.

7. Ribeiro F, et al. Feridas e úlceras cutâneas: Lisboa, Grafilarte; 1999.

8. Liang J. Self-reported physical health among aged adults. J Gerontol 1986; 41: 248-60.

9. WHO. The role of PA in healthy aging gene. 1998.

10. Braden B. Risk assessement in pressure ulcer prevention. Chronic wound care. Health Management Publications, 1997.

11. Folstein M, Folstein S, McHugh P. MiniMental state: a practical method for grading the cognitive state of patients for the clinician. J Psychiatr Res 1975; 12: 189-98.
12. Sun G, French C, Martin G et al. Comparison of multifrequency bioelectrical impedance analysis with dual-energy $\mathrm{X}$-ray absorptiometry for assessment of percentage body fat in a large, healthy population. Am J Clin Nutr 2001; 81: 74-88.

13. Bottoni A, Oliveira G, Ferrini M, Waitzberg D. Avaliação nutricional: exames laboratoriais. In: Waitzberg DL (editor). Nutrição oral, enteral e parenteral na prática clínica. 3. ed. São Paulo: Atheneu; 2000. p.279-94.

14. Jeejeebhoy K. Nutritional assessment. Clin Nutr 1998; 27: 347-69.

15. Defloor T, Grypdonck M. Pressure ulcers: validation of two risk assessment scales. J Clin Nurs 2004; 14: 373-82.

16. Young T. Classificação das ùlceras de pressão: Nursing 1997; 9: 21.

17. Kunding J. Medição do volume de feridas. Nursing 1996; 98.

18. PRESSURE ULCERS IN ADULTS: prediction and prevention. Washington, DC: US Department of Health and Human Services; 1992.

19. Nes M, Herrmann F, Gold G, Michel J, Rizzoli R. Does the mini nutritional assessment predict hospitalization outcomes in older people? Age Ageing 2001; 30: 221-6.

20. THE MANAGEMENT OF PRESSURE ulcers in primary and secondary care: a clinical practice guideline 20 Cavendish Square London W1G ORN; 2005. 
21. Armstrong D., Bortz P. An integrative review of pressure relief in surgical patients: AORN J 2001; 73: 645-8.

22. Lyder C, Preston J, Grady J, Scinto J, Allman R. Quality of care for hospitalized medicate patients at risk for pressure ulcers. Arch Intern Med 2001; 161: 1549-54.

23. Pereira A, Freitas D, Mata E, Baeta L, Pereira M. Incidência das úlceras por pressão. S. 1.: Serviço de Medicina Dois Mulheres do Centro Hospitalar do Funchal; 2000.

24. Duarte C, Canto M, Garcias'T, Silva G, Jesus J. Epidemiologia das úlceras por pressão na região autónoma da Madeira. S.l.: s. ed.; 2001.

25. Dealey C. The care of wounds. a guide for nurses. Oxford: Blacwell Scientific Publications; 1999.
26. Perneger T, Gaspoz J, Rae A, Borst F, Heliot C. Contribution of individual items to the performance of the Norton pressure ulcer prediction scale: Geneva: University Hospitals; 2003.

27. Bryant R, et al. Acute and chronic wounds: nursing management. 2nd ed. Missouri, 2000.

28. Ferry M, Alix E. A nutrição da pessoa idosa. Lisboa: Lusociência; 2004. 394 p.

29. Christensson I, Unossom M, Ek A. Evaluation of nutritional assessment techniques in elderly people admitted to municipal care. Eur J Clin Nutr 2002; 56: 810-8.

30. Naváez P, Fernández M. Úlceras por presión. Evaluation de un protocolo: Rev ROL Enfermería 1997; 20: 73-80.

31. Fernandes L. Uma revisão integrativa da literatura. São Paulo: 2000. 